\title{
Metformin use associated with protective effects for ocular complications in patients with type 2 diabetes - observational study
}

\author{
Sanita Maleškićn ${ }^{1}$ Jasna Kusturica ${ }^{1}$, Edis Gušić ${ }^{2}$, Maida Rakanović-Todić ${ }^{1}$ \\ Damir Šečić ${ }^{3}$, Lejla Burnazović-Ristić ${ }^{1}$, Aida Kulo ${ }^{1}$
}

${ }^{1}$ Department of Pharmacology and Toxicology, School of Medicine University of Sarajevo, Sarajevo, Bosnia and Herzegovina, ${ }^{2}$ Department of Health Care, Ministry of Internal Affairs of Canton Sarajevo, Sarajevo, Bosnia and Herzegovina, ${ }^{3}$ Department of Pathophysiology, School of Medicine University of Sarajevo, Sarajevo, Bosnia and Herzegovina

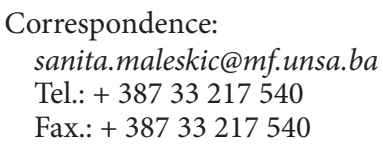

Received: 15 June 2017

Accepted: 30 November 2017

Key words: Type 2 diabetes - Metformin • Ocular complications.

\begin{abstract}
Objective. The aim was to study the association of the use of an oral antihyperglycemic agent metformin with the presence of ocular complications in patients with type 2 diabetes (T2D). Methods. Medical records were reviewed for 234 patients with diagnosed T2D. $81.2 \%$ $(n=190)$ patients were using metformin and $18.8 \%(n=44)$ using other oral antihyperglycemic agents. Plasma glucose concentration, glycated haemoglobin, and the presence of ocular complications in patients treated with metformin were compared to those in patients treated with other oral antihyperglycemic agents. Results. Ocular complications occurred in 65 patients $(27.8 \%)$. Patients treated with metformin had fewer ocular complications compared to patients treated with other oral antihyperglycemic agents $\left(\chi^{2}=19.985 ; \mathrm{p}<0.0001\right)$. After adjustment for gender, age, duration of T2D, serum concentration of cholesterol, smoking, body mass index and presence of other diseases, treatment with metformin decreased the odds of both glaucoma $(\mathrm{OR}=0.14$, $95 \%$ CI: $0.03-0.57, p=0.006)$ and diabetic retinopathy $(\mathrm{OR}=0.33,95 \%$ CI: $0.14-0.82, p=0.017)$ compared with other oral antihyperglycemic agents. Conclusion. Our results suggest that metformin may have a protective effect on ocular complications, especially glaucoma, in patients with T2D. The effects of metformin either regarding prevention of ocular complications or ocular complications already developed in patients with T2D, should be further investigated.
\end{abstract}

\section{Introduction}

Type 2 diabetes (T2D), as a chronic systemic metabolic condition, causes changes to the eyes, leading to ocular complications such as glaucoma, diabetic retinopathy, cataracts and other ocular lesions $(1,2)$. This has been proven to be one of the major causes of visual impairment in these patients (3).

A relationship between T2D and glaucoma has been described (4). It is confirmed that diabetes mellitus (DM) is an etiological factor for neovascular glaucoma, but there are still controversial opinions about its association with open-angle glaucoma or angle-closure glaucoma (5). Factors identified to increase the risk of glaucoma in patients with T2D are corneal stiffness, corneal hysteresis and enlargement of the optic cup, recognized as an important morphological feature of the glaucomatous eye (6). However, the age of the patient is a major risk factor for the onset of glaucoma (5). 
Diabetic retinopathy is the most common ocular complication of DM and, if not treated, may lead to vision loss (7). Factors that affect the occurrence of diabetic retinopathy are the duration of DM, the severity of hyperglycaemia and the age of the patient (8). Diabetic retinopathy is also related to hypertension, dyslipidaemia, pregnancy, nephropathy, and anaemia (6). Inflammation-mediated pathways and angiogenesis are thought to underlie the progression of diabetic retinopathy $(9,10)$. Due to its anti-inflammatory, antiangiogenic and antiaging activity, the oral anti-hyperglycaemic agent metformin could possibly exercise a protective mechanism by inhibiting neovascularization in diabetic retinopathy, as well as in other retinopathies (11-13). Some studies have documented a less known but also important mechanism of metformin, the reduction of ischemia that may underlie the beneficial effects of metformin in ischemia-related retinopathy $(10,14)$. However, some recent studies have described the proangiogenic effects of metformin that could favour diabetic retinopathy $(14,15)$.

Numerous studies have documented the association between T2D and cataracts (5). Patients with T2D are more likely to have cataracts diagnosed at an earlier age, even before any diabetic retinopathy is seen (16, 17). The suggested mechanisms that may precipitate the formation of diabetic cataracts are: increased osmotic stress caused by activation of the polyol pathway, nonenzymatic glycation of lens proteins and deposition of its end-products in the lens, and increased oxidative stress $(5,18,19)$. Based on the risk factors for ocular complications in patients with T2D on the one hand, and the effects of metformin on the other, the potential protective effects of metformin treatment for ocular complications could be hypothesized. However, the data regarding this are controversial and limited to a few studies $(2,20-24)$.
We aimed to study the association of the use of the oral anti-hyperglycaemic agent metformin with the presence of ocular complications in patients with T2D.

\section{Patients and methods}

\section{Study design and data collection}

This was an observational study. Patients with T2D treated at the Sarajevo Public Institution Health Centre in the period between 1997 and 2014 were screened for inclusion in this study. Out of 500 records reviewed, patients with confirmed diagnosis of T2D and who were treated with the same oral antihyperglycemic agents for at least one year, were included in the study $(\mathrm{n}=234$, $46.8 \%)$. Patients treated with insulin and patients without complete medical records were not included in the study. Data were collected on the age of patients, gender, duration of $\mathrm{T} 2 \mathrm{D}$, body mass index (BMI), the medication used in the treatment of T2D, smoking habits, plasma glucose concentration, glycated haemoglobin (HbAlc), serum cholesterol concentration, the presence of ocular complications and the presence of other diseases at the last check-up.

\section{Ethics statement}

The study was approved by Local Ethics Committee organized at the School of Medicine of the University of Sarajevo. All procedures were performed in accordance with the ethical standards of the institutional and/ or national research committee, the 1964 Helsinki Declaration and its later amendments, or comparable ethical standards.

\section{Statistical analysis}

Upon completion of the data collection, data were analysed using IBM SPSS Statistics software (Statistical Package for Social Sci- 
ences, SPSS Inc, Chicago, Illinois, USA) version 20.0. Continuous numerical variables with normal distribution were expressed as mean \pm standard deviation, while those not normally distributed were expressed as median and interquartile range. Depending on the type of distribution of continuous numerical variables, a comparison between two treatment groups was made either by using the unpaired Student's t-test or the Mann-Whitney test as appropriate. Comparison between patients with different ocular complications was performed using ANOVA. The Chi-square test was used to determine the relationship between categorical variables. The univariate associations between the presence of ocular complications and metformin treatment were assessed with logistic regression. For multivariate analysis, models were adjusted for gender, age, duration of T2D (model II) and additionally for serum cholesterol concentrations, smoking, BMI and the presence of other diseases (model III). The Bonferroni adjustment at $\alpha=0.05 / 3=0.017$ was applied. To assess whether the findings were influ- enced by the older age of some patients, a sensitivity analysis was performed by excluding the patients in the highest tertile of age ( $>70$ years). P-values less than 0.05 were considered statistically significant.

\section{Results}

This study included 234 patients with diagnosed T2D. For at least one year, $81.2 \%$ $(n=190)$ of the patients were treated with only metformin, and $18.8 \%(n=44)$ of the patients were treated with only one of other oral antihyperglycemic agents, either sulfonylureas (glibenclamide, glimepiride, gliclazide) or thiazolidinediones (pioglitazone). Patients treated with metformin were more likely to be women, younger, with shorter duration of T2D and with fewer other comorbidities compared to the patients treated with other oral antihyperglycaemic agents. The subjects' characteristics are presented in Table 1.

For the plasma glucose concentration measured at the last check-up, the unpaired $t$-test showed a marginally significant difference with slightly higher plasma glucose con-

Table 1 Patients characteristics

\begin{tabular}{|c|c|c|}
\hline \multirow{2}{*}{ Parameters } & \multicolumn{2}{|l|}{ Patients treated with } \\
\hline & Metformin $(n=190)$ & Other *AHG agents $(n=44)$ \\
\hline Age, years & $65.6 \pm 10.5$ & $72.1 \pm 12.4$ \\
\hline Gender (male), n (\%) & $86(45.3)$ & $25(56.8)$ \\
\hline Duration of ${ }^{\mathrm{T}} \mathrm{T} 2 \mathrm{D}$ (years) & $5.0(2-11)$ & $8.0(4-11)$ \\
\hline${ }^{\ddagger} \mathrm{BMI}$ & $29.9 \pm 5.07$ & $28.6 \pm 4.31$ \\
\hline Smoking, n (\%) & $87(45.8)$ & $20(45.5)$ \\
\hline Serum concentration of cholesterol $(\mathrm{mmol} / \mathrm{l})$ & $5.6 \pm 1.29$ & $5.1 \pm 1.35$ \\
\hline Plasma glucose concentration (mmol/l) & $8.2(6.9-9.3)$ & $7.4(6.8-8.4)$ \\
\hline${ }^{\mathrm{s}} \mathrm{HbA} 1 \mathrm{c}(\%)$ & $7.2(6.8-8.8)$ & $7.0(6.7-7.9)$ \\
\hline Presence of other diseases, $\mathrm{n}(\%)$ & $182(95.8)$ & $44(100)$ \\
\hline \multicolumn{3}{|l|}{ Ocular complications } \\
\hline Glaucoma, n (\%) & $6(3.2)$ & $5(11.4)$ \\
\hline Diabetic retinopathy, n (\%) & $11(5.8)$ & $6(13.6)$ \\
\hline Cataract, n (\%) & $24(12.6)$ & $13(29.5)$ \\
\hline
\end{tabular}

Data are presented as mean \pm standard deviation or median (interquartile range) or percentages. *Antihyperglycemic; ${ }^{\dagger}$ Type 2 diabetes; ${ }^{*}$ Body Mass Index; ${ }^{\S}$ Glycated hemoglobin. 
centrations in patients treated with metformin, compared to patients treated with other oral antihyperglycemic agents $(8.7 \pm 4.5 \mathrm{mmol} / \mathrm{L}$ vs. $7.7 \pm 1.9 \mathrm{mmol} / \mathrm{L} ; \mathrm{p}=0.05 ; 95 \% \mathrm{CI}:-1.36$ $0.01)$. The median HbAlc value in the metformin group was $7.2 \%$ and in the other treatment group 7.0\%. The Mann-Whitney U-test showed no significant difference between the two groups $(\mathrm{p}=0.179)$. The one-way ANOVA test showed no significant difference in plasma glucose concentrations $[F(3,230)=0.236$, $\mathrm{p}=0.871]$ and $\mathrm{HbAlc}$ levels $[\mathrm{F}(3,230)=0.669$, $\mathrm{p}=0.572]$ between patients with different ocular complications. The median HbA1c levels in patients with different ocular complications are presented in Figure 1.

Out of 234 patients with diagnosed T2D, ocular complications occurred in 65 patients (27.8\%). Patients treated with metformin had fewer ocular complications compared to patients treated with other oral antihyper- glycemic agents ( $\mathrm{c} 2$ test $=19.985 ; \mathrm{p}<0.0001$ ) (Table 1). The association of T2D therapy with ocular complications are shown in Table 2. When the association between metformin use and the presence of ocular complications was tested, metformin use was associated with fewer ocular complications in an unadjusted model. The odds ratio of ocular complications in patients treated with metformin $(\mathrm{OR}=0.23,95 \%$ CI: 0.12-0.46, $\mathrm{p}<0.001)$ remained significant after adjustment for gender, age and duration of T2D (OR $=0.22,95 \%$ CI: $0.10-0.49, \mathrm{p}<0.001)$ and also after adjustment for gender, age, duration of $\mathrm{T} 2 \mathrm{D}$, serum cholesterol concentration, smoking, $\mathrm{BMI}$ and the presence of other diseases $(\mathrm{OR}=0.19$, 95\% CI: 0.08-0.42, $\mathrm{p}<0.0001$ ).

Next, the association between metformin use and each ocular complication was tested. Table 2 shows that the odds ratio of glaucoma was significant in unadjusted models

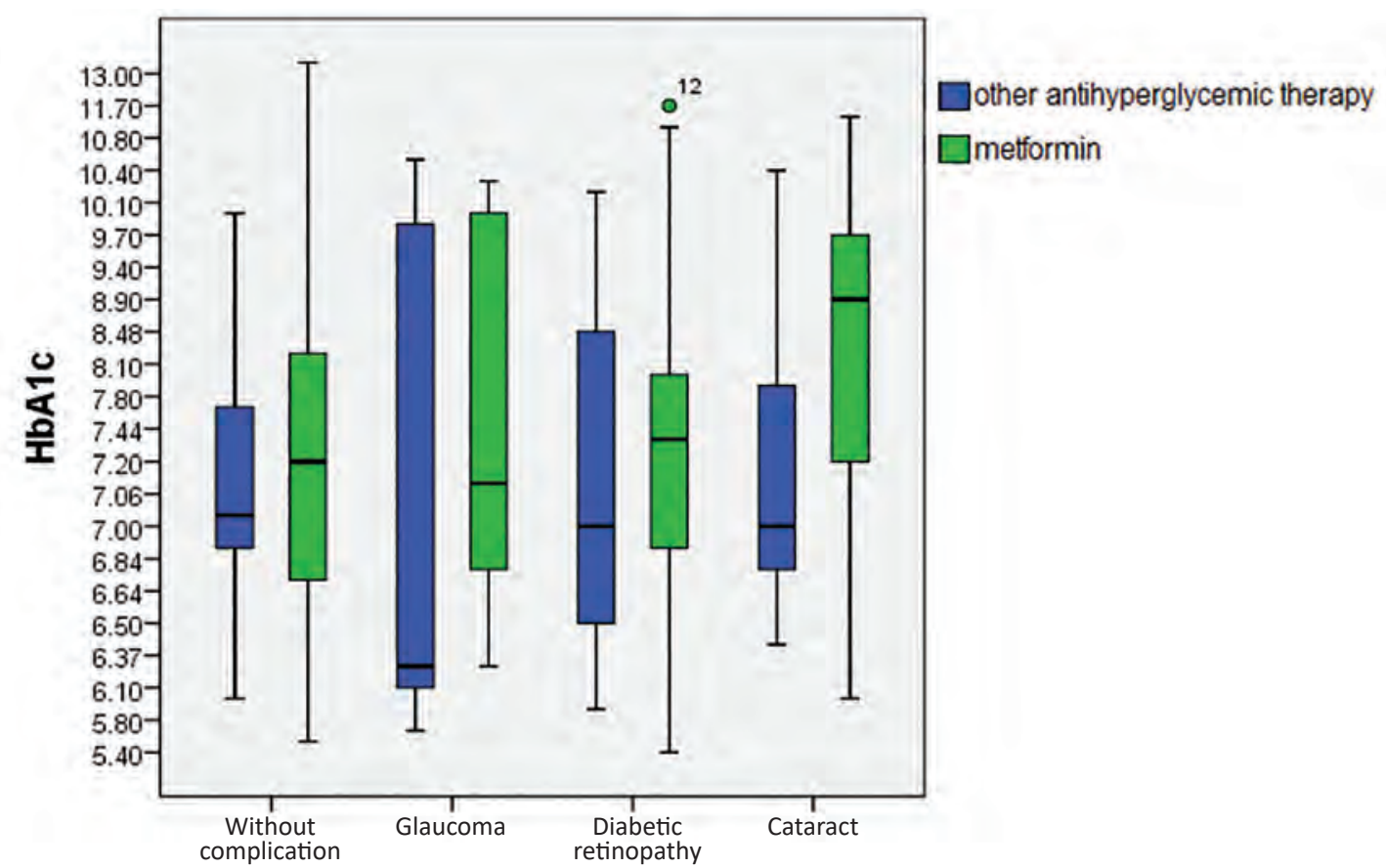

Figure 1 Glycated haemoglobin (HbA1c) levels in patients from the two treatment groups with different ocular complications. The solid horizontal line denotes the median value of $\mathrm{HbA} 1 \mathrm{c}$ in two treatment groups with different ocular complications; the box represents the $25^{\text {th }}$ and $75^{\text {th }}$ interquartile range. The whisker represents the minimum and maximum value. 
Table 2 Metformin therapy and ocular complications in patients with T2D

\begin{tabular}{lllllll}
\hline \multirow{2}{*}{ Outcome } & \multicolumn{7}{l}{ Metformin therapy } & & & \\
\cline { 2 - 7 } & Model I & & Model II & Model III & \\
\cline { 2 - 7 } & OR $(95 \% \mathrm{Cl})$ & $\mathrm{p}$ & $\mathrm{OR}(95 \% \mathrm{Cl})$ & $\mathrm{P}$ & $\mathrm{OR}(95 \% \mathrm{Cl})$ & $\mathrm{P}$ \\
\hline Ocular complications & $0.23(0.12,0.46)$ & $<0.001$ & $0.22(0.10,0.49)$ & $<0.001$ & $0.19(0.08,0.42)$ & $<0.0001$ \\
Glaucoma & $0.25(0.07,0.88)$ & 0.030 & $0.21(0.06,0.78)$ & 0.020 & $0.14(0.03,0.57)$ & 0.006 \\
Diabetic retinopathy & $0.35(0.16,0.75)$ & 0.007 & $0.40(0.17,0.94)$ & 0.037 & $0.33(0.14,0.82)$ & 0.017 \\
Cataract & $0.39(0.14,1.12)$ & 0.079 & $0.36(0.10,1.26)$ & 0.11 & $0.40(0.10,1.52)$ & 0.18 \\
\hline
\end{tabular}

T2D= Type 2 diabetes; Model I: Univariate analysis; Model II: Analysis adjusted for gender, age and duration of T2D; Model III: Analysis adjusted for gender, age, duration of T2D, serum concentration of cholesterol, smoking, BMI and presence of other diseases; Predictor: Metformin use other therapy was used as a reference category.

$(\mathrm{OR}=0.25,95 \% \mathrm{CI}: 0.07-0.88, \mathrm{p}=0.030)$ and also in two adjusted models (OR $=0.21,95 \%$ CI: $0.06-0.78, p=0.020$; $\mathrm{OR}=0.14,95 \% \mathrm{CI}$ : $0.03-0.57, \mathrm{p}=0.006)$. It is also shown that the odds ratio of diabetic retinopathy in patients treated with metformin $(\mathrm{OR}=0.35,95 \% \mathrm{CI}$ : $0.16-0.75, p=0.007)$ remained significant after adjustment for gender, age and duration of T2D (OR=0.40, 95\% CI: 0.17-0.94, $\mathrm{p}=0.037)$ and also after adjustment for gender, age, duration of $\mathrm{T} 2 \mathrm{D}$, serum cholesterol concentration, smoking, BMI and the presence of other diseases (OR $=0.33,95 \% \mathrm{CI}$ : $0.14-0.82, \mathrm{p}=0.017$ ).

The association of metformin use and the presence of cataracts was also analysed. The odds ratio of cataracts was not significant for metformin use in either unadjusted or adjusted models. The results remained significant after Bonferroni adjustment at $\alpha=0.05 / 3=0.017$. In sensitivity analysis, the highest tertile of age ( $>70$ years) was excluded and the results remained significant for overall ocular complications $(\mathrm{OR}=0.184$, 95\% CI: 0.05-0.67, $\mathrm{p}=0.010$ ) and glaucoma $(\mathrm{OR}=0.10,95 \% \mathrm{CI}: 0.01-0.91, \mathrm{p}=0.041)$.

\section{Discussion}

Although with no significant differences in the control of the HbA1c levels and the duration of T2D, and with a marginally significant difference in fasting plasma glucose concentrations between the two treatment groups, patients with $\mathrm{T} 2 \mathrm{D}$ treated with metformin presented with less glaucoma, diabetic retinopathy, and cataracts. This suggests the possible protective effect of metformin treatment against these ocular complications in patients with T2D. Besides the duration of DM, glycaemic control is recognized as one of the strongest predictors for the development of diabetic retinopathy $(25,26)$. However, our study showed that patients treated with metformin had a lower incidence of ocular complications, although the control of plasma glucose concentrations and $\mathrm{HbAlc}$ levels were not within the recommended range for most of the patients in both treatment groups.

The micro- and macro-vascular complications of T2D lead to morbidity and reduced life expectancy $(2,27,28)$. Use of metformin as calorie restriction (CR) mimetic drug has been shown to be associated with reduced risk of late-onset eye diseases such as glaucoma, but also diabetic retinopathy, cataract and macular degeneration (20). In this regard, our study showed that the prevalence of glaucoma was lower among patients treated with metformin compared to patients treated with other oral anti-hyperglycaemic agents (3.2\% v. $11.4 \%)$. Although the relationship between DM and glaucoma was until recently controversial, new studies have identified an association between these 
two disorders (4). Glaucoma is a type of neuropathy and DM could cause optic neuropathy. In addition, it has also been found that the central cornea is thicker in patients with DM, and this could also cause higher intraocular pressure (5). To our knowledge, only one retrospective cohort study has shown that metformin use is associated with a reduced risk of developing openangle glaucoma and the authors suggested that metformin could have an impact on glaucoma risk on multiple levels, including glycaemic control, but also as a CR mimetic drug $(20,21)$. As previous studies suggested that age-related tissue changes significantly contribute to glaucoma development (29), the need has already been postulated to explore whether metformin, as a CR mimetic drug with an anti-aging effect, could delay progression of tissue damage (13).

Similar to our study, Richards et al. found that glaucoma risk reduction in a population with DM was dose-dependent for metformin and independent of glycaemic control (21). They also found that other anti-hyperglycaemic agents did not result in a similar glaucoma risk reduction (21). New prospective clinical trials are necessary to evaluate whether metformin might also have protective effects on the occurrence of glaucoma in non-diabetic patients. Such findings could lead to novel treatment approaches and better glaucoma control.

Our study showed the possible beneficial protective role that metformin may have for diabetic retinopathy, compared to other antihyperglycemic agents ( $13.6 \%$ vs. $5.8 \%$ patients). This protective effect of metformin on diabetic retinopathy has also been shown in an animal study (10). Moreover, our results are consistent with the results from Ryu et al. (22) who found that $45.5 \%$ of patients from the non-metformin group developed diabetic retinopathy compared to $27.3 \%$ of the patients from the group treated with metformin. Additionally, the United Kingdom
Prospective Diabetes Study (UKPDS) Group showed a significantly lower $(\mathrm{p}=0.044)$ rate of progression of retinopathy in overweight patients with intensive blood-glucose control, treated with metformin, compared to those treated with other treatments (diet or intensive treatment with chlorpropamide, glibenclamide, or insulin) (2).

\section{Limitations of the study}

The total number of patients included in the study was small, and therefore replication in a larger study is needed. Also, as data on the onset or duration of ocular complications were not available, we cannot conclude whether patients had any complications at the moment when the therapy started, with either metformin or other oral antihyperglycemic agents.

\section{Conclusion}

The results of our study suggest that metformin use might be associated with protective effects for ocular complications in patients with T2D. As this is an observational study and a causal/protective relationship cannot be established, the effects of metformin either on the prevention of ocular complication or on ocular complications already developed in patients with T2D should be further investigated through large prospective clinical trials.

\footnotetext{
What is already known on this topic

Diabetes-associated ocular complications are often associated with inflammation-mediated pathways, angiogenesis, and agerelated tissue changes. Besides its effects on glycaemic control, metformin has also been shown to have anti-inflammatory, antiangiogenic and calorie restriction related anti-aging activity. There are very limited data suggesting the protective role of metformin treatment against glaucoma and retinopathy in patients with type 2 diabetes.
}

What this study adds

This observational study confirmed the protective effects of metformin treatment against glaucoma and diabetic retinopathy, and suggested its protective effects on cataracts in patients with type 2 diabetes. 
Authors' contributions: Conception and design: SM, JK and EG; Acquisition, analysis and interpretation of data: JK and AK; Drafting the article: SM, JK and AK; Revising it critically for important intellectual content: MRT, DŠ and LBR; Approved final version of the manuscript: SM, JK and AK.

Conflict of interest: The authors declare that they have no conflict of interest.

\section{References}

1. Bolen S, Feldman L, Vassy J, Wilson L, Yeh HC, Marinopoulos S, et al. Systematic review: Comparative effectiveness and safety of oral medications for type 2 diabetes mellitus. Ann Intern Med. 2007;147(6):386-99.

2. UK Prospective Diabetes Study (UKPDS) Group. Effect of intensive blood-glucose control with metformin on complications in overweight patients with type 2 diabetes (UKPDS 34). Lancet. 1998;352(9131):854-65.

3. Signorini AM, Fondelli C, Renzoni E, Puccetti C, Gragnoli G, Giorgi G. Antioxidant effects of gliclazide, glibenclamide, and metformin in patients with type 2 diabetes mellitus. Curr Ther Res. 2002;63(7):411-20.

4. Zhou M, Wang W, Huang W, Zhang X. Diabetes Mellitus as a Risk Factor for Open-Angle Glaucoma: A Systematic Review and Meta-Analysis. PLoS One. 2014;9(8):e102972.

5. Sayin N, Kara N, Pekel G. Ocular complications of diabetes mellitus. World J Diabetes. 2015;6(1):92108.

6. Guedes G, Tsai JC, Loewen NA. Glaucoma and Aging. Curr Aging Sci. 2011;4(2):110-7.

7. Frank RN. Diabetic retinopathy. N Engl J Med. 2004;350:48-58.

8. Del Cañizo Gómez FJ, Fernández Pérez C, Moreno Ruiz I, de Gorospe Pérez-Jáuregui C, Silveira Rodríguez B, González Losada T, et al. Microvascular complications and risk factors in patients with type 2 diabetes. Endocrinol Nutr. 2011;58(4):1638.

9. Fong DS, Aiello L, Gardner TW, King GL, Blankenship G, Cavallerano JD, et al. Retinopathy in diabetes. Diabetes Care. 2004;27(1):84-7.

10. Kern TS. Contributions of inflammatory processes to the development of the early stages of diabetic retinopathy. Exp Diabetes Res. 2007;95103.

11. Maruthur NM, Tseng E, Hutfless S, Wilson LM, Suarez-Cuervo C, Berger Z, et al. Diabetes Medications as Monotherapy or Metformin-Based
Combination Therapy for Type 2 Diabetes. Ann Intern Med. 2016;164(11):740-51.

12. Joe SG, Yoon YH, Choi JA, Koh J-Y. Anti-Angiogenic Effect of Metformin in Mouse OxygenInduced Retinopathy Is Mediated by Reducing Levels of the Vascular Endothelial Growth Factor Receptor Flk-1. PLoS ONE. 2015;10(3):e0119708.

13. Anisimov VN. Metformin: Do we finally have an anti-aging medication? Cell Cycle. 2013;12(22):3483-9.

14. Joussen AM, Poulaki V, Mitsiades N, Cai WY, Suzuma I, Pak J, et al. Suppression of Fas-FasLinduced endothelial cell apoptosis prevents diabetic blood-retinal barrier breakdown in a model of streptozotocin-induced diabetes. FASEB J. 2003;17(1):76-8.

15. Dallaglio K, Bruno A, Cantelmo AR, Esposito AI, Ruggiero L, Orecchioni S, et al. Paradoxic effects of metformin on endothelial cells and angiogenesis. Carcinogenesis. 2014;35(5):1055-66.

16. Seck T, Nauck M, Sheng D, Sunga S, Davies MJ, Stein PP, et al. Safety and efficacy of treatment with sitagliptin or glipizide in patients with type 2 diabetes inadequately controlled on metformin: a 2-year study. Int J Clin Pract. 2010;64(5):562-76.

17. Pascolini D, Mariotti SP. Global estimates of visual impairment: 2010. Br J Ophthalmol. 2012;96(5):614-8.

18. Jeganathan VS, Wang JJ, Wong TY. Ocular association of diabetes other than diabetic retinopathy. Diabetes Care. 2008;31(9):1905-12.

19. Pirie A. Epidemiological and biochemical studies of cataract and diabetes. Invest Ophthalmol. 1965;4:629-37.

20. Lin HC, Stein JD, Nan B, Childers D, NewmanCasey PA, Thompson DA, et al. Association of Geroprotective Effects of Metformin and Risk of Open-Angle Glaucoma in Persons With Diabetes Mellitus. JAMA Ophthalmol. 2015;133(8):915-23.

21. Richards JE, Lin HC, Nan B, Talwar N, Childers D, Newman-Casey PA, et al. Targeting aging: Geroprotective Medication Metformin Reduces Risk of Adult-onset Open-angle Glaucoma. Invest Ophthalmol Vis Sci. 2014;55(13):1668.

22. Ryu C, Munie M, Noorulla S, Edwards P, Qiao X, Gao H. Effect of Metformin on the Development of Diabetic Retinopathy. Invest Ophthalmol Vis Sci. 2013;54(15):2449.

23. Kim AJ, Chang JY, Shi L, Chang RC, Ko ML, Ko GY. The Effects of Metformin on Obesity-Induced Dysfunctional Retinas. Invest Ophthalmol Vis Sci. 2017;58(1):106-18.

24. Diabetes Prevention Program Research Group. Long-term effects of lifestyle intervention or met- 
formin on diabetes development and microvascular complications over 15-year follow-up: the Diabetes Prevention Program Outcomes Study. Lancet Diabetes Endocrinol. 2015;3(11):866-75.

25. Fowler JM. Microvascular and Macrovascular Complications of Diabetes. Clin Diabetes. 2008;26(2):77-82.

26. Bennett WL, Maruthur NM, Singh S, Segal JB, Wilson LM, Chatterjee R, et al. Comparative effectiveness and safety of medications for type 2 diabetes: an update including new medications and 2-medication combinations. Ann Intern Med. 2011;154(9):602-13.
27. Grimaldi A, Grangé V, Allannic H, Passa P, Rodier $\mathrm{M}$, Cornet $\mathrm{P}$, et al. Epidemiological analysis of patients with Type 2 diabetes in France. J Diabetes Complications. 2000;14(5):242-9.

28. Zhang X, Zhao J, Zhao T, Liu H. Effects of intensive glycemic control in ocular complications in patients with type 2 diabetes: a meta-analysis of randomized clinical trials. Endocrine. 2015;49(1):7889.

29. Guedes G, Tsai JC, Loewen N. Glaucoma and Aging. Curr Aging Sci. 2011;4(2):110-7. 